\title{
El Reduccionismo de Carnap
}

\author{
Oscar Augusto Garcia Zarate \\ Departamento Académico de Filosofia
}

$\mathbf{S}$

i bien Rudolf Camap no fue fundador del Círculo de Viena, se convirtió en su representante más destacado. Por sus grandes aportes a la lógica, a la semántica y a la epistemología, su presencia constituye una figura fuerte no sólo de la filosofia contemporánea sino de la universal. La filosofia -aun en la era pospositivista- lo valora como exponente de una de sus tradiciones más profundas.

La base teórica del neopositivismo lo constituyen fundamentalmente las siguientes tesis: la negación de la metafisica, el fisicalismo y la unidad de las ciencias, y la verificabilidad empírica. Pero más que de una teoria se trataba de un programa a realizar. De hecho, el empirismo lógico estrecho y rígido de la primera época se fue abriendo y matizando cada vez más hasta desembocar en diversas corrientes de filosofla analftica.

Su método

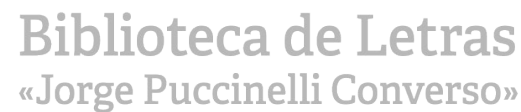

Su método de análisis lógico se inscribe y desarrolla dentro del marco filosófico del positivismo lógico y en el marco general de la filosofia analf́tica que historicamente lo precede y lo sobrevive. A través de este método, Carnap se proponía eliminar del lenguaje de la ciencia todos los enunciados que no fueran verificables ${ }^{1}, y$, con esto, librarlo de los enunciados de la metaffsica, de la ética normativa y de la axiología; de los enunciados modales, los enunciados del

En el contexto de los planteamientos del positivismo lógico se postulo, tomando como referencia la postura asumida por Wittgenstein. que la imposibilidad logica de verificar un enunciado indicaba que éste o bien constituía un sinsentido o pseudoproposición, esto es, un absurdo, o bien que, simplemente, carecia de sentido, aunque sin ser por esto un sinsentido. Como se sabe, esta substancial diferencia ya habla sido seftalada de modo claro por Wittgenstein, que en el Tractarus Logico-Philosophicus habla de la carencia de sentido de una proposición (simnlos) y de sinsentido o absurdo (unsinning). para referirse a las proposiciones lógicas y a las pseudoproposiciones, respectivamente. Cf. Tractalus Logico-Philosophicus, p. 87, aforísmo 4.461 y 4.4611 ; p. 73, aforismo 4.1272 . 
'modo material de hablar' y, en general, de todos los pseudoenunciados y sinsentidos, depurándolo para que sólo conservara enunciados contingentes, los cuales, una vez verificados, serían verdaderos o falsos. Asi, el análisis lógico del lenguaje permitiría identificar los errores estructurales que se dan en su ámbito, y que generan los extravíos que Carnap encuentra, por ejemplo, en la metafísica. Según esto, su método no sería otra cosa que una pesquisa a nivel sintáctico.

\section{La hipótesis del fisicalismo}

En consonancia con este planteamiento, Carnap, siguiendo el programa del positivismo lógico relativo a la búsqueda y construcción de la unidad de la ciencia, bajo la hipótesis de que «toda oración con sentido es reductible al lenguaje fisico», sostiene que la hipótesis del fisicalismo "para toda oración psicológica hay una oración física correspondiente», es en realidad simplemente un problema de sintaxis, de lógica. Consecuentemente, el problema del fisicalismo es un problema de lenguaje. Asi, sobre el particular escribe:

... de si para toda oracion psicblógica, $\mathrm{S}_{1}$, hay o no una oración física correspondiente, $\mathrm{S}_{2}$, equipolente a $\mathrm{S}_{1}$. La tesis del fisicalismo responde afirmativamente a esta pregunta pero, como es natural, esta posición está siempre abierta a discusión si se le oponen objeciones. El problema del fisicalismo es un problema científico o, más exactamente, lógico, sintáctico y sólo se podrá solventar mediante ulteriores consideraciones y debates. Mas el problema de si, usando et modo material, hemos de hablar de dos estados diferéntes, uno mental otro fisić, o de uno solo, no es más que un problema de decisión acerca del uso del lenguaje, una cuestión de gusto, por así decir. No se trata de una cuestión de hecho, como creen los metafísicos en sus disputas ${ }^{2}$.

De esta manera, la psicología se reduciría a la biología, y ésta a la física; es decir, reduciendo toda la explicación científica a las leyes de la física en última instancia, todas las leyes resultarían ser meramente leyes resultantes o aditivas.

\section{Una impugnación del Reduccionismo}

El enfoque reduccionista de la ciencia que tiene en Rudolf Carnap a uno de sus más connotados defensores constituye, a estas alturas y como señala $\mathrm{Pa}$ -

2 CARnap, R. Filosofia y sintaxis logica, p. 317. 
trick Suppes, "un llanto nostálgico" por la unidad de la ciencia. En atención a esto, consideramos pertinente en este punto traer a colación el análisis que en relación con este aspecto efectúa este destacado epistemólogo en un artículo publicado como parte de una antologia suya titulada Estudios de filosofia $y$ metodologia de la ciencia.

Suppes asume que la postura reduccionista es abordable desde tres perspectivas, a saber, reducción del lenguaje, reducción del objeto de estudio y reducción del método.

En lo que atañe al reduccionismo en relación con el lenguaje de la ciencia, Suppes sostiene que éste no es pasible de ser unificado, pues la cada vez más acusada divergencia de lenguajes que se opera en el ámbito cientifico en virtud de la creciente tecnificación de aquéllos, determina un panorama plural irreductible, con respecto al cual la idea de una unificación sustancial del lenguaje científico resulta poco menos que quimérica.

En cuanto a la reducción del objeto de estudio, Suppes -tomando como eje la concepción atomista que se proyecta desde los lejanos tiempos de la antigua Grecia, a partir de las ideas de Demócrito-considera que este modo de concebir la estructura primordial de la realidad, y que lleva a suponer, en ultima instancia, que el objeto de estudio, sea cual fuere su forma, es susceptible de ser reducido a átomos en movimiento, ha perdido vigencia definitivamente en base a los descubrimientos de la fisica cuantica đue conducen a la concepción de la realidad como compuesta de particulas que se siguen dividiendo, de modo que resulta incierto referirse a entidades últimas al estilo del reduccionismo fuerte que formulara Demócrito, pues dichas particulas están hechas, al parecer, de enjambres de "algo", que no se sabe bien qué es.

Una situación similar se presenta en el ámbito de las matemáticas, pues la reducción de una gran parte de éstas a la teoría de conjuntos constituye una estéril formalidad, toda vez que se ha llegado a advertir que los fundamentos de las mismas no poseen un grado de certeza absoluto, de modo que, más bien, los desarrollos que se han dado a partir de aquéllos han sido mejor comprendidos. Teniendo en cuenta esto, un intento de reducción no tendria sentido.

Respecto al reducionismo en función del método, pareciera que éste es el aspecto que brinda mayores posibilidades de plantear una efectiva reductibilidad de la ciencia, y esto en base a la consideración de que ésta apela al uso de 
métodos matemáticos en todos sus dominios. Sin embargo, como señala Suppes, esta aparente unidad de método no va muy lejos, pues el verdadero meollo del asunto lo constituye el aspecto experimental del método, aspecto bajo el cual si se verifica una pluralidad de procedimientos de acuerdo a la ciencia de que se hable; incluso esta heterogeneidad metodológica se presenta al interior de una misma ciencia, según se trate de uno u otro dominio especializado.

De esta manera, a partir del cuestionamiento del enfoque reduccionista de la ciencia, Suppes propone el reemplazo de esta postura por otra que tome en cuenta la pluralidad irreductible de métodos, lenguaje y objetos de estudio que el actual panorama de la ciencia nos muestra. Consecuentemente, concluye que el programa reduccionista de Carnap ha fracasado y, actualmente, es insostenible ninguna forma de reduccionismo:

Parte de mi tesis sobre la pluralidad de la ciencia es que los lenguajes de diferentes campos de la ciencia son divergentes más que convergentes en la medida en que se vuelven cada vez más técnicos ${ }^{3}$.

La consecuencia más importante de este análisis es que la filosofía quedaba reducida a su mínima expresión, es decir, simplemente a un método, al método del análisis lógico o análisis sintáctico del lenguaje de la ciencia; o en otras palabras, se reduciría a la lógica, a la lógica aplicada, o "lógica de la ciencia", como dice Carnap o, como también dice, a la "epistemología". La citas que se detallan confirmāiestädeduceiónde Letras

La filosofia "Jata láciencia sólo desde el punto de vista lógico, la filosofia es lógica de la ciencia, esto es, análisis lógico de los conceptos, proposiciones, pruebas y teorías de la ciencia ${ }^{4}$.

La única tarea propia de la filosofía es el análisis lógico, por lo que el problema principal, que tenemos que resolver es: ¿Qué es el análisis lógico? $?^{5}$

...todas las tesis y problemas de la filosofia pertenecen a la sintaxis lógica. El único método de la filosofia es el método de la sintaxis lógica,

3 Suppes, Patrick. (1988). Estudios de filosofia y metodologia de la ciencia. Madrid, Alianza Editorial, pp. 78-84.

4 Carnap, R. "Sobre el carácter de los problemas filosóficos", p. 26.

5 Carnap, R. Filosofia y sintaxis lógica, p. 305. 
es decir, del análisis de la estructura formal del lenguaje como sistema de reglas 6 .

Pero entonces, ¿qué le queda a la filosofia si todas las proposiciones que afirman algo son de naturaleza empirica y pertenecen por tanto a la ciencia fáctica? Lo que queda no son proposiciones, no es una teoria ni un sistema, sino exclusivamente un método, esto es, del análisis lógico?.

Aparte de las cuestiones de las ciencias individuales, únicamente las cuestiones del antálisis lógico de la ciencia, de sus oraciones, términos, conceptos, teorías, etc., se dejan como cuestiones cientificas genuinas. Llamaremos a este complejo de cuestiones la lógica de la ciencia ... la lógica de la ciencia toma el lugar de la inextricable maraña de problemas que se conoce como filosofia".

Quien quiera que comparta con nosotros el punto de vista antimetafisico, concordará por ello en que todos los problemas filosóficos que tienen sentido pertenecen a la sintaxis?.

De esta manera, según Carnap, quedarian eliminadas todas las demás ramas de la filosofia reconocidas y consideradas desde hace siglos, tales como la ética, estética, axiologia, gnoseologia, ontología y, obviamente, la metafisica. Aunque nada impide al filósofo desempeñar una función útil analizando y esclareciendo los conceptos que figuran en el empleo del lenguaje diario, es en el empleo científico del lenguaje que principalmente se desarrolla su labor, puesto que éste se consideraba el más importante.

En esta perspectiva, por ejemplo, los problemas del espacio, tiempo, causa, efecto, entre otros, se reducen simple y llanamente a problemas de lenguaje y de lógica. En efecto, Carnap escribe:

... las oraciones de la filosofia -incluso después de haber eliminado la metafísica- parecen referirse no sólo a la forma de las expresiones lingulisticas, sino también, y tal vez principalmente, a otros objetos tales como a la estructura del espacio y el tiempo, a las relaciones entre causa

" Ibid., p. 335.

- Carnap. R. "La superación de lia metafisica a través del andílisis lógico del lenguaje", p. 84.

- Carnap, R. The Logical Symtax of Languqje, p. 279.

"Ibid., p. 280 , 
y efecto, a las relaciones reales entre lo físico y lo mental, al carácter del número y de las funciones numéricas, a la necesidad, a la contingencia, a la posibilidad o imposibilidad de las condiciones, y cosas por el estilo. Habremos de mostrar más tarde que las oraciones fillosóficas de estos tipos parecen, por su apariencia engañosa, referirse a los objetos mencionados, cuando en realidad sólo se refieren a formas lingüísticas ${ }^{10}$.

Consecuentemente, los físicos y los matemáticos no estarian capacitados para estudiar la naturaleza, estructura y demás propiedades del espacio y el tiempo; los biólogos, los neurólogos, los psicólogos y los especialistas en cibernética e inteligencia artificial no podrian estudiar el cerebro y lo mental; los matemáticos, los lógicos y los filósofos de las matemáticas estarían imposibilitados para estudiar la naturaleza y el carácter de los números y funciones; finalmente, en general, los filósofos, lógicos y epistemólogos verian vedada la posibilidad de estudiar la relación entre causa y efecto, necesidad, posibilidad o imposibilidad, contingencia, etc., porque todos estos temas se reducirian únicamente a problemas de formas de lenguaje, a problemas de sintaxis lógica, de análisis lógico. Pero, ¿es esto plausible? Ya, en 1956, J.O. Urmson decia:

La filosofia no puede ser sólo sintaxis lógica, ni puede ser caracterizada un lenguaje como una lista de trazos sobre el papel con reglas de formación y de transformación. Carnap, y sus compañeros hicieron todo lo que puede hacerse con estå tesisł́

"Jorge Puccinelli Converso"

Por nuestra parte no sólo pensamos que la filosofia 'no puede ser', sino que afirmamos categóricamente que la filosofia no es -como nunca lo fue tampoco antes del positivismo lógico-sólo sintaxis lógica.

Y, actualmente, $\dot{i}$ alguien sigue a Carnap y cree en su reduccionismo, en su filosofía de la ciencia, que posteriormente los críticos han denominado la «concepción heredada», por haber sido legada por el positivismo lógico, del que Carnap fue uno de los representantes más preclaros y consecuentes? Pues ya no, nadie le cree ni le sigue seriamente; asimismo, ya nadie cree en el programa del positivismo lógico, como dice Frederick Suppe:

\footnotetext{
10. Carnap R. Filosofia y sintaxis lógica, p. 317.

"URmson, J.O. El análisis filosófico, p. 150.
} 
Para concluir, virtualmente todo el programa del positivismo para la filosofia de la ciencia ha sido repudiado por la filosofia de la ciencia contemporánea ... El positivismo hoy día realmente pertenece a la historia de la filosofia de la ciencia ${ }^{12}$.

En nuestra opinión, la concepción de ciencia, metafisica y filosofia de Camap es completamente obsoleta y errada, pues así como Popper en su momento trazara una linea de demarcación entre ciencia y aquello que no era ciencia, en la actualidad se reconoce la inexistencia de una solución de continuidad entre ciencia y metafísica, esto es, no hay demarcación absoluta entre ambas, pues en tanto que filosofia y ciencia son dos formas de saber, hay entre ellas un continuum, de modo tal que la línea de frontera que supuestamente las separa se hace difusa.

Para mostrar la inexistencia de una línea de demarcación entre la ciencia y la filosofía es suficiente con indicar dos cosas. En primer lugar, la constatación de que muchos problemas considerados históricamente como filosóficos devinieron posteriormente en científicos. En segundo lugar, la existencia de problemas que son al mismo tiempo filosóficos y cientificos, es decir, problemas de intersección entre la ciencia y la filosofia.

Ilustramos esta aseveración con los siguientes ejemplos de problemas que fueron considerados filosóficos pero, una vez que fueron resueltos pasaron, en consecuencia, al campo dela ciencia:En fisica se creia que: a) La materia consistia de cuatro elementos tierra agual, airey fuego; b) En el universo cada cosa tenía su lugar natural; los astros y todo el cielo estaban hechos de una materia divina. En biologia se creía que todas las especies animales aparecieron $-o$ fueron creadas- en el estado en que se conocen actualmente. En psicologia : a) Hipócrates creía que la conducta humana se explicaba en función de cuatro liquidos o "humores" que hay en el cuerpo: sangre, bilis amarilla, bilis negra y flema; b) Descartes creía que había ideas innatas, por ejemplo, la idea de "dios". En ciencias sociales se creía que la esclavitud, la propiedad privada y la existencia de ricos y pobres eran naturales.

Podemos, asimismo, considerar otros ejemplos de problemas de carácter filosófico-científico, es decir, de intersección entre la ciencia y la filosofia: a) ¿Es

12 Suppes, Frederick The Structure of Scientific Theories, p. 632. 
la materia divisible infinitamente? b) ¿Es posible crear máquinas que piensan? c) ¿Son el tiempo y el espacio divisibles infinitamente?

Mario Bunge precisa en cuanto a la demarcación entre ciencia y filosofia:

Yo nunca la he hecho. Justamente, a diferencia de los positivistas y de Popper, yo sostengo, e intento probar, que no hay fronteras entre las dos. $\mathrm{Y}$ esto porque a) toda investigación científica supone principios filosófi$\cos y, b)$ toda filosofía que se pretenda científica recoge resultados de la investigación científica. No, para mi no hay fronteras entre ambos campos. La ciencia se solapa parcialmente con la filosofia ${ }^{13}$.

Están actualmente debilitadas las teorías de la significación y de la verdad que Carnap basó en la verificación -sea en los enunciados protocolarios o en último término en el fisicalismo-, por el planteamiento de los problemas sobre la referencia y la elección de las teorias que hizo Quine. Se les reconoce un carácter fundamental en la epistemología contemporánea, según este aporte de Quine, a la inescrutabilidad de la referencia y a la infradeterminación de las teorias.

Según la inescrutabilidad de la referencia no existe ninguna relación necesaria y univoca entre el lenguaje - por ejemplo, las teorías científicas-y la realidad; es decir, no hay manera alguna de establecer una relación necesaria y unívoca, válida para todas las culturas y todas las épocas, entre los sustantivos o nombres y sus referentes. De ahtque, segünetpunfo de vista actual, la única manera de hacer ciencia y plantear teorías es adoptando una posición convencionalista $\mathrm{y}$, por ende, pragmatista.

De aquí surge directamente el problema de la infradeterminación de las teorias, es decir el problema de elegir qué teoría es la única válida, pues frente a un mismo conjunto de datos empíricos es posible plantear varias que igualmente den cuenta o expliquen dichos hechos. Esta discusión, iniciada en los trabajos de Quine "Dos dogmas del empirismo", "Palabra y objeto", "Relatividad ontológica" y "Raices de la referencia", se ha desarrollado en los trabajos de epistemólogos contemporáneos, como Larry Laudan y F. Suppes, entre otros.

${ }^{13}$ Bunge, Mario (1999). Vigencia de la filosofia. Lima, Fondo Editorial de la UIGV, pp. 49-50. 
Finalmente, también cabe una observación a la concepción carnapiana de la lógica. Él nos dice en The Logical Syntax of Language, hablando acerca de la sintaxis lógica de cualquier lenguaje dentro del contexto de la lógica extensional:

El carácter lógico de todas las oraciones de estos lenguajes es, entonces, invariante en relación a desplazamientos espacio-temporales; dos oraciones del mismo lenguaje tendrán el mismo carácter independienternente de dónde, cuándo o por quién fueron habladas. En el caso de oraciones que tienen dependencia extrasintáctica, esta invariante puede ser obtenida por medio de la adición de designaciones de persona, lugar, y tiempo ${ }^{14}$.

Carnap estaba animado por una fe única en la lógica clásica. Prácticamente la consideraba todopoderosa y casi perfecta -como Kant acerca de la lógica aristotélica-, razón por la cual no quería admitir ninguna otra distinta, por ejemplo, la lógica modal. Ahora, con la profusión de un sinnúmero de lógicas no clásicas, la concepción carnapiana de la lógica ha quedado obsoleta.

Bastaría sefialar, $y$ esto a modo de conclusión, que en última instancia, el reduccionismo en que desemboca la postura asumida por Carnap adquiere un marcado carácter cientificista. En efécto, la nueva lógica, como él la denomina ${ }^{25}$, adoptada como instrumental metodológico a emplearse en el análisis del lenguaje, así como la postulación del ideal de la ciencia unificada, esto es, la plasmación de un único lenguaje -el lenguaje fisicalista-, concebido como la expresión más acabada en el que deberia traducirse cualquier discurso que pretendiere poseer el estatuto de científico, constituyer el binomio en que se expresa de modo cabal tal reduccionismo.

4 Carnap, R. The Logical Symtax of Language, p. 232.

is Carmap, R (1930). Lo antigua y la mueva lógica, p. 19. 